\title{
The particular solutions of some types of Euler-Cauchy ODE using the differential transform method
}

\author{
Meshari Alesemi, M. A. El-Moneam*, Bader S. Bader, E. S. Aly \\ Mathematics Department, Faculty of Science, Jazan University, Jazan, Kingdom of Saudi Arabia.
}

\begin{abstract}
In this paper, we apply the differential transform method to find the particular solutions of some types of Euler-Cauchy ordinary differential equations. The first model is a special case of the nonhomogeneous $n^{\text {th }}$ order ordinary differential equations of Euler-Cauchy equation. The second model under consideration in this paper is the nonhomogeneous second order differential equation of Euler-Cauchy equation with a bulge function. This study showed that this method is powerful and efficient in finding the particular solution for Euler-Cauchy ODE and capable of reducing the size of calculations comparing with other methods.
\end{abstract}

Keywords: Differential equations, differential transform method, Euler-Cauchy equations.

2010 MSC: 65L05.

(C2019 All rights reserved.

\section{Introduction}

There are more than one method to solve the Euler-Cauchy equation such as the Laplace transform, the variation of parameters method, and the method of reduction of the order. Kim [13] applied the Laplace transform to find the solution of a homogeneous Euler-Cauchy equation of the second order ODE, Abualrab [1], found a solution of a special case of nonhomogeneous Euler-Cauchy equation using the variation of parameters, and Haarsa and Pothat [14], found the solution of a second order Euler-Cauchy equation with bulge function using the reduction of the order method. The differential transform method presented by Pukhov [12] in 1982 and the concept of differential transform proposed first by Zhou [23] in 1986 when applied to solve linear and nonlinear initial value problems in electric circuit analysis. The differential transform method presented as a new method based on Taylor series [23] and considered as a semi-analytical technique uses the Taylor series to construct the solutions of differential equations in the form of a power series. The method represents an iterative procedure for obtaining analytic series solutions of differential equations and useful for obtaining exact and approximate solutions of linear ordinary differential equations [7] and system of linear ordinary differential equations [20]. In this paper,

\footnotetext{
*Corresponding author

Email addresses: malesem@jazanu.edu.sa (Meshari Alesemi), maliahmedibrahim@jazanu.edu.sa (M. A. El-Moneam), bsaad1757@gmail.com (Bader S. Bader), elkhateeb@jazanu.edu.sa (E. S. Aly)
}

doi: $10.22436 /$ jnsa.012.03.02

Received: 2018-07-21 Revised: 2018-08-09 Accepted: 2018-09-22 
the differential transform method has been applied to the second order nonhomogeneous ODE of EulerCauchy equation with a bulge function and the general nonhomogeneous Euler-Cauchy equation. The differential transform method develops from the differential equation with initial conditions a recurrence relation that finally leads to the solution of algebraic equations as coefficients of Taylor's series solution. Moreover, the DT method does not evaluate the derivatives symbolically and this gives advantages over other methods. For the related work, (see [18, 19, 21, 22]). The paper is written in the following structure. In Section 2 we introduce the concept of a one dimension differential transform and review some basic fundamental theorems from references, and then we prove a lemma which is very important in our work. The differential transform method to find the particular solution of special cases of the nonhomogeneous ODE of Euler-Cauchy equation is presented in Section 3. We conclude the paper with some remarks in Section 4.

\section{The differential transform method}

In this section, we introduce the concept of one-dimensional differential transform and review some basic fundamental theorems [8-17]. We assume that the function $f(x) \in C^{\infty}(I)$, and $x_{0}$ be any point of I. Then the Taylor's series of $f(x)$ about $x_{0}$ can be written as

$$
f(x)=\sum_{k=0}^{\infty} \frac{1}{k !} \frac{d^{k} f(x)}{d x^{k}}\left(x-x_{0}\right)^{k} .
$$

Definition 2.1. Let the function $f(x)$ be analytical function about $x_{0}=0$, then the $k^{\text {th }}$ differential transform of $f(x)$ is defined by

$$
\mathrm{F}(\mathrm{k})=\mathrm{D}_{\mathrm{T}}\{\mathrm{f}(\mathrm{x})\}=\frac{1}{\mathrm{k} !}\left[\frac{\mathrm{d}^{\mathrm{k}} \mathrm{f}(\mathrm{x})}{\mathrm{d} \mathrm{x}^{\mathrm{k}}}\right]_{\mathrm{x}=0} .
$$

Note that, the inverse differential transform of $F(k)$ is defined by

$$
\mathrm{D}_{\mathrm{T}^{-1}}\{\mathrm{~F}(\mathrm{k})\}=\mathrm{f}(\mathrm{x})=\sum_{\mathrm{k}=0}^{\infty} \mathrm{F}(\mathrm{k}) \mathrm{x}^{\mathrm{k}} .
$$

Theorem $2.2([1-11,13-17])$. Let $\mathrm{f}(\mathrm{x})$ and $\mathrm{g}(\mathrm{x})$ be analytic functions, with differential transforms $\mathrm{F}(\mathrm{k})$ and $\mathrm{G}(\mathrm{k})$, respectively, then for $\sigma$ and $\beta$ :

1. $\mathrm{D}_{\mathrm{T}}\{\sigma f(x)+\beta g(x)\}=\sigma \mathrm{F}(\mathrm{k})+\beta \mathrm{G}(\mathrm{k})$.

2. $D_{\mathrm{T}}\left\{\frac{\mathrm{d}^{\mathrm{n}} \mathrm{f}(\mathrm{x})}{\mathrm{d} \mathrm{x}^{\mathrm{n}}}\right\}=\frac{(\mathrm{k}+\mathrm{n}) !}{\mathrm{k} !} \mathrm{F}(\mathrm{k}+\mathrm{n})$.

3. $\mathrm{D}_{\mathrm{T}}\left\{\mathrm{x}^{\mathrm{m}}\right\}=\mathrm{F}(\mathrm{k})=\delta_{\mathrm{k}, \mathrm{m}}=\delta(\mathrm{k}-\mathrm{m}) \frac{(\mathrm{k}+\mathrm{n}) !}{\mathrm{k} !} \mathrm{F}(\mathrm{k}+\mathrm{n})$, where $\delta_{\mathrm{k}, \mathrm{m}}$ is the Kronecker delta.

4. If $\mathrm{f}(\mathrm{x})=\int_{0}^{\mathrm{x}} \mathrm{g}(\mathrm{x}) \mathrm{d} \mathrm{x}$, then the differential transform of the function $\mathrm{f}(\mathrm{x})$ is given by be $\mathrm{F}(\mathrm{k})=\frac{\mathrm{G}(\mathrm{k}-1)}{\mathrm{k}}$.

5. If $f_{1}(x)$ and $f_{2}(x)$ be analytic functions, with $f(x)=f_{1}(x) \cdot f_{2}(x)$, then $F(k)=\sum_{n=0}^{k} F_{1}(n) F_{2}(k-n)$.

6. $\mathrm{D}_{\mathrm{T}}\left\{x^{m_{f}}(\mathrm{n})(x)\right\}=\sum_{i=0}^{k} \delta_{i, m} \frac{(k+n-i) !}{(k-i) !} \mathrm{F}(k+n-i)$, and if $m=n$, then $D_{T}\left\{x^{n} f^{(n)}(x)\right\}=\prod_{i=0}^{n-1}(k-$ i) $F(k)$.

7. Let $f(x)=\alpha^{\alpha x}$, then $F(k)=\frac{\alpha^{k}}{k !}$.

8. $\mathrm{D}_{\mathrm{T}}\left\{e^{\alpha x_{f}(n)}(x)\right\}=\sum_{i=0}^{k} \frac{\alpha^{i}}{i !} \frac{(k+n-i) !}{(k-i) !} F(k+n-i)$. 
9. $\mathrm{D}_{\mathrm{T}}\{\cos (\alpha x)\}=\frac{\alpha^{\mathrm{k}}}{\mathrm{k} !} \cos \left(\frac{\mathrm{k} \pi}{2}\right)$ and $\mathrm{D}_{\mathrm{T}}\{\sin (\alpha x)\}=\frac{\alpha^{\mathrm{k}}}{\mathrm{k} !} \sin \left(\frac{\mathrm{k} \pi}{2}\right)$.

10. Let $\mathrm{f}(\mathrm{x})$ be analytic function, with $\mathrm{D}_{\mathrm{T}}\{\mathrm{f}(\mathrm{x})\}=\mathrm{F}(\mathrm{k})$, then $\mathrm{D}_{\mathrm{T}}\left\{\frac{\mathrm{d}}{\mathrm{dx}}\left(\mathrm{f}^{(\mathrm{n})}(\mathrm{x})\right)\right\}=\frac{(\mathrm{k}+1)(\mathrm{k}+\mathrm{n}) !}{\mathrm{k} !} \mathrm{F}(\mathrm{k}+\mathrm{n})$. Note that for $\mathrm{n}=1$ then $\mathrm{D}_{\mathrm{T}}\left\{\frac{\mathrm{d}}{\mathrm{dx}}\left(\mathrm{xf}^{(1)}(\mathrm{x})\right)\right\}=(\mathrm{k}+1)^{2} \mathrm{~F}(\mathrm{k}+1)$.

11. Let $\mathrm{f}(\mathrm{x})$ be analytic function with $\mathrm{D}_{\mathrm{T}}\{\mathrm{f}(\mathrm{x})\}=\mathrm{F}(\mathrm{k})$, then $\mathrm{D}_{\mathrm{T}}\left\{\frac{\mathrm{d}}{\mathrm{dx}}\left(\mathrm{x}^{\mathrm{m}} \mathrm{f}^{(\mathrm{n})}(\mathrm{x})\right)\right\}=\frac{(\mathrm{k}+1)(\mathrm{k}+\mathrm{n}-\mathrm{m}+1) !}{(\mathrm{k}-\mathrm{m}+1) !} \mathrm{F}(\mathrm{k}+$ $n-m+1)$.

12. The differential transform $\mathrm{F}(\mathrm{k})$ for any analytic nonlinear term $\mathrm{f}(\mathrm{y})$ can be calculated from the formula $F(k)=\frac{1}{k !} \frac{d^{k}}{d \mu^{k}}\left[\sum_{i=0}^{\infty} Y(i) \mu^{i}\right], k \geqslant 0$, where $Y(k)$ is the differential transform of $y(x)$.

13. $\mathrm{D}_{\mathrm{T}}\{\mathrm{tf}(\mathrm{y})\}=\mathrm{F}(\mathrm{k}-1)$, where $\mathrm{F}(\mathrm{k})$ is the differential transform of $\mathrm{f}(\mathrm{y})$.

14. $D_{\mathrm{T}}\left\{\mathrm{t}^{2} \mathrm{f}(\mathrm{y})\right\}=\mathrm{F}(\mathrm{k}-2)$, where $\mathrm{F}(\mathrm{k})$ is the differential transform of $\mathrm{f}(\mathrm{y})$.

15. $\mathrm{D}_{\mathrm{T}}\left\{\mathrm{t}^{\mathrm{m}} \mathrm{f}(\mathrm{y})\right\}=\mathrm{F}(\mathrm{k}-\mathrm{m})$, where $\mathrm{F}(\mathrm{k})$ is the differential transform of $\mathrm{f}(\mathrm{y})$.

\section{Lemma 2.3.}

1. One have for $z(t)=t \frac{d y}{d t}, z(k)=k Y(k)$, and

2. for $z(\mathrm{t})=\mathrm{t}^{2} \frac{\mathrm{d}^{2} \mathrm{y}}{\mathrm{dt}^{2}}, z(\mathrm{k})=\mathrm{k}(\mathrm{k}-1) \mathrm{Y}(\mathrm{k})$, and

3. for $z(t)=t^{3} \frac{d^{3} y}{d^{3}}, z(k)=k(k-1)(k-2) Y(k)$, and

4. for $z(t)=t^{n} \frac{d^{n} y}{d t^{n}}, z(k)=k(k-1) \cdots(k-n+2) Y(k)$.

Proof. (1) Let $u(t)=\frac{d y}{d t} \longrightarrow U(k)=(k+1) Y(k+1)$. Then or $z=t u(t) \longrightarrow z(k)=u(k-1)=k Y(k)$.

(2) Let $u(t)=\frac{d^{2} y}{d t^{2}} \longrightarrow u(k)=(k+2)(k+1) Y(k+2)$. Then for $z=t^{2} u(t) \longrightarrow z(k)=u(k-2)=$ $k(k-1) Y(k)$. The proof of (3) and (4) is similar to (1) and (2).

\section{Euler-Cauchy equation}

In this section, we apply the differential transform method (DTM) to find the particular solution of special cases of nonhomogeneous Euler-Cauchy ordinary differential equation.

The general $\mathrm{n}^{\text {th }}$ order Cauchy-Euler ODE is defined as follows

$$
a_{n} t^{n} \frac{d^{n} y}{d t^{n}}+a_{n-1} t^{n-1} \frac{d^{n-1} y}{d t^{n-1}}+\cdots+a_{1} t \frac{d y}{d t}+a_{0}=r(t)
$$

As a first special case, let

$$
r(t)=b t^{m},
$$

where, $m \geqslant n$ and $m$ must not equal any of the roots of the characteristic equation and $m, n$ are integer, we have the following formula for the particular solution of (3.1).

Lemma 3.1. The particular solution of the $\mathrm{n}^{\text {th }}$ order Euler-Cauchy ODE (3.1), where $\mathrm{m} \geqslant \mathrm{n}$ and $\mathrm{m}$ must not equal any of the roots of the characteristic equation and $\mathrm{m}, \mathrm{n}$ are integer can be expressed by

$$
y(t)=\sum_{i=0}^{\infty} \frac{b \delta(k-m) t^{k}}{a_{n} \prod_{i=0}^{n-1}(k-i)+a_{n-1} \prod_{i=0}^{n-2}(k-i)+\cdots+a_{2} k(k-1)+a_{1} k+a_{0}} .
$$

Proof. Apply the differential transform to both sides of equation (3.1), and making use of Lemma 2.3, we get

$$
a_{n} \prod_{i=0}^{n-1}(k-i) Y(k)+a_{n-1} \Pi_{i=0}^{n-2}(k-i) Y(k)+\cdots+a_{2} k(k-1) Y(k)+a_{1} k Y(k)+a_{0} Y(k)=b \delta(k-m),
$$

where $\delta(k-m)$ is the $k^{\text {th }}$ differential transform of $t^{m}$. 
From (3.2), we have the recurrence relation

$$
Y(k)=\frac{b \delta(k-m)}{a_{n} \Pi_{i=0}^{n-1}(k-i)+a_{n-1} \Pi_{i=0}^{n-2}(k-i)+\cdots+a_{2} k(k-1)+a_{1} k+a_{0}} .
$$

Applying Definition 2.1, we get the formula

$$
y(t)=\sum_{i=0}^{\infty} \frac{b \delta(k-m) t^{k}}{a_{n} \prod_{i=0}^{n-1}(k-i)+a_{n-1} \prod_{i=0}^{n-2}(k-i)+\cdots+a_{2} k(k-1)+a_{1} k+a_{0}} .
$$

Example 3.2. Consider the third order ODE of Cauchy-Euler equation

$$
t^{3} y^{\prime \prime \prime}-3 t^{2} y^{\prime \prime}+6 t y^{\prime}-6 y=5 t^{4}
$$

Solution. By using equation (3.3), we get

$$
Y(k)=\frac{5 \delta(k-m)}{k(k-1)(k-2)-3 k(k-1)+6 k-6} .
$$

From the definition of $\delta(k-m), m=4$, we get, $Y(4)=\frac{5}{6}, Y(k)=0, k \neq 4$. Therefore, the particular solution is given by (3.4) as follows

$$
y(t)=\frac{5}{6} t^{4}
$$

Example 3.3. Consider the Euler-Cauchy equation

$$
t^{2} y^{\prime \prime}-t y^{\prime}-3 y=2 t^{2}
$$

Solution. From the recurrence relation (3.3), we get

$$
Y(k)=\frac{2 \delta(k-m)}{k(k-1)-k-3} .
$$

From the definition of $\delta(k-m), m=2$, we get, $Y(2)=-\frac{2}{3}, Y(k)=0, k \neq 2$. Therefore, the particular solution will be given by (3.4) in the form

$$
y(t)=\frac{-2}{3} t^{2}
$$

Example 3.4. Consider the Euler-Cauchy equation

$$
t^{2} y^{\prime \prime}-2 t y^{\prime}+2 y=t^{3}
$$

Solution. Using the recurrence relation (3.3), we get

$$
Y(k)=\frac{\delta(k-m)}{k(k-1)-2 k+2} .
$$

From the definition of $\delta(k-m), m=3$, we get, $Y(3)=\frac{1}{2}, Y(k)=0, k \neq 3$, therefore, the particular solution will be given by (3.4) as

$$
y(t)=\frac{1}{2} t^{3} .
$$

Example 3.5. Consider the second order ODE of Cauchy-Euler equation

$$
t^{2} y^{\prime \prime}-4 t y^{\prime}+4 y=t^{2}
$$


Solution. Applying the recurrence equation (3.3), yields

$$
Y(k)=\frac{\delta(k-m)}{k(k-1)-4 k+4}
$$

From the definition of $\delta(k-m), m=2$, we get, $Y(2)=-\frac{1}{2}, Y(k)=0, k \neq 2$, therefore, the particular solution will be given by (3.4) as

$$
y(t)=-\frac{1}{2} t^{2}
$$

Example 3.6. Consider the Euler-Cauchy ODE

$$
\mathrm{t}^{2} \mathrm{y}^{\prime \prime}+10 \mathrm{ty} \mathrm{y}^{\prime}+8 \mathrm{y}=\mathrm{t}^{2}
$$

Solution. By applying the recurrence relation (3.3), we have

$$
Y(k)=\frac{\delta(k-m)}{k(k-1)+10 k+8} .
$$

From the definition of $\delta(k-m), m=2$, we get, $Y(2)=\frac{1}{30}, Y(k)=0, k \neq 2$, therefore, the particular solution will be given by (3.4) as

$$
y(t)=\frac{1}{30} t^{2}
$$

As the second special case, we consider the second order Euler-Cauchy differential equation with

$$
r(t)=e^{-\frac{(t-l)^{2}}{2}} \text {. }
$$

Lemma 3.7. The particular solution of the nonhomogeneous Euler-Cauchy ODE with a bulge function

$$
t^{2} y^{\prime \prime}+a t y^{\prime}+b y=e^{-\frac{(t-l)^{2}}{2}},
$$

where $\mathrm{a}, \mathrm{b}$ are constants, $\mathrm{l}$ is a positive constant, and $\mathrm{y}(\mathrm{t})$ is unknown function can be expressed by

$$
Y(k)=\sum_{k=2}^{\infty} \frac{F(k)}{k[a+(k-1)]+b} t^{k},
$$

where $F(k)$ is the $k^{\text {th }}$ differential transform of the bulge function $f(t)=e^{-\frac{(t-l)^{2}}{2}}$.

Proof. Applying the differential transform on both sides of equation (3.5), and making use of lemma 2.3, we get

$$
k(k-1) Y(k)+a k Y(k)++b Y(k)=F(k) .
$$

From equation (3.6), we get the recurrence relation

$$
Y(k)=\frac{F(k)}{k[a+(k-1)]+b^{\prime}}
$$

where, $F(k), k=2,3,4,5,6$, are given by Definition 2.1 as follows

$$
\begin{aligned}
& F(2)=\left(-\frac{1}{2}+\frac{l^{2}}{2}\right) e^{-\frac{1}{2} l^{2}}, \quad F(3)=\left(-\frac{l}{2}+\frac{l^{3}}{3 !}\right) e^{-\frac{1}{2} l^{2}} \\
& F(5)=\left(\frac{l}{8}-\frac{l^{3}}{12}+\frac{l^{5}}{5 !}\right) e^{-\frac{1}{2} l^{2}}, \quad F(6)=\left(-\frac{1}{48}+\frac{l^{2}}{16}-\frac{l^{4}}{48 !}+\frac{l^{6}}{6 !}\right) e^{-\frac{1}{2} l^{2}}, \\
& \mathrm{~F}(4)=\left(\frac{1}{8}-\frac{l^{2}}{4}+\frac{l^{4}}{4 !}\right) e^{-\frac{1}{2} l^{2}}
\end{aligned}
$$

Therefore the particular solution of (3.5) is given by

$$
y(t)=\sum_{k=2}^{\infty} Y(k) t^{k}=\sum_{k=2}^{\infty} \frac{F(k)}{k[a+(k-1)]+b} .
$$




\section{Conclusion}

Based on the work has been done, it can be concluded that the differential transform method can be used to find the particular solutions of some types of Euler-Cauchy ODE accurately. The recurrence relations are very simple; therefore, they are easy to compute. This study showed that the differential transform technique is capable of reducing the size of calculations comparing with the method of reduction of order and the method of variation of parameters.

\section{References}

[1] M. S. Abdualrab, A formula for solving a special case of Euler-Cauchy ODE., Int. Math. Forum, 4 (2009), 1997-2000. 1, 2.2

[2] J. Ali, One dimensional differential transform method for some higher order boundary value problems in finite domain, Int. J. Contemp. Math. Sci., 7 (2012), 263-272.

[3] M. T. Alquran, Applying differential transform method to nonlinear partial differential equations: a modified approach, Appl. Appl. Math., 7 (2012), 155-163.

[4] A. Aslanov, Determination of convergence intervals of the series solutions of EmdenFowler equations using polytropes and isothermal spheres, Phy. Lett. A, 372 (2008), 3555-3561.

[5] K. Batiha, B. Batiha, A new algorithm for solving linear ordinary differential equations, World Appl. Sci. J., 15 (2011), 1774-1779.

[6] C. Bervillier, Status of the differential transformation method, Appl. Math. Comput., 218 (2012), 10158-10170.

[7] J. Biazar, M. Eslami, Differential transform method for quadratic Riccati differential equation, Int. J. Nonlinear Sci., 9 (2010), 444-447. 1

[8] S.-H. Chang, I.-L. Chang, A new algorithm for calculating one dimensional differential transform of nonlinear functions, Appl. Math. Comput., 195 (2008), 799-805. 2

[9] E. A. Elmabrouk, F. Abdewahid, Useful Formulas for One-dimensional Differential Transform, Britsh J. Appl. Sci. Tech., 18 (2016), 1-8.

[10] V. S. Ertürk, Application of differential transformation method to linear sixth-order boundary value problems, Appl. Math. Sci. (Ruse), 1 (2007), 51-58.

[11] V. S. Ertürk, Approximate Solutions of a Class of Nonlinear Differential Equations by Using Differential Transformation Method, Int. J. Pure Appl. Math., 30 (2006), 403-407. 2.2

[12] G. G. Ev Pukhov, Differential transforms and circuit theory, Circuit Theory Appl., 10 (2008), 265-276. 1

[13] B. Ghil, H. Kim, The Solution of Euler-Cauchy Equation Using Laplace Transform, Int. J. math. Anal., 9 (2015), $2611-2618$. $1,2.2$

[14] P. Haarsa, S. Pothat, The Reduction of Order on Cauchy-Euler Equation with a Bulge Function, Appl. Math. Sci., 9 (2015), 1139-1143. 1

[15] I. H. A. H. Hassan, V. S. Ertürk, Solution of differential types of the linear and nonlinear higher-order boundary value problems by differential transformation method, Eur. J. Pure Appl. Math., 2 (2009), 426-447.

[16] K. Parand, Z. Roozbahani, F. Bayat Babolghani, Solving nonlinear Lane-Emden type equations with unsupervised combined artificial neural networks, Int. J. Industrial Mathematics, 5 (2013), 12 pages.

[17] M. A. Soliman, Y. Al-Zeghayer, Aproximate analytical solution for the isothermal Lane Emden equation in a spherical geometry, Revist Mexicanade Astronmiay Atrofisca, 15 (2015), 173-180. 2, 2.2

[18] A.-M. Wazwas, A new algorithm for solving differential equations of LaneEmden type, Applied Math. Comput., 118 (2001), 287-310. 1

[19] A.-M. Wazwas, The modified decomposition method for analytic treatment of differential equations, Appl. Math. Comput., 173 (2006), 165-176. 1

[20] A. Yildrim, T. Özis, Solutions of singular IVPs of LaneEmden type by the variational iteration method, Nonlinear Anal., 70 (2009), 2480-2484. 1

[21] E. M. E. Zayed, M. A. El-Moneam, Some oscillation criteria for second order nonlinear functional ordinary differential equations, Acta Math. Sci. Ser. B (Engl. Ed.), 27 (2007), 602-610. 1

[22] E. M. E. Zayed, S. R. Grace, H. El-Metwally, M. A. El-Moneam, The oscillatory behavior of second order nonlinear functional differential equations, Arab. J. Sci. Eng. Sect. A Sci., 31 (2006), 23-30. 1

[23] J. K. Zhou, Differential transformation and its applications for electrical circuits, Huarjung University Press, wuuhahn, (1986). 1 